\title{
Oral antivirals for the prevention and treatment of SARS-CoV-2 infection
}

Vicente Soriano ${ }^{*}$, Carmen de-Mendoza², Benson Edagwa ${ }^{3}$, Ana Treviño ${ }^{1}$, Pablo Barreiro ${ }^{4}$, José V. Fernandez-Montero ${ }^{5}$, and Howard E. Gendelman ${ }^{3}$

${ }^{1}$ UNIR Health Sciences School \& Medical Center, Madrid, Spain; ${ }^{2}$ Department of Internal Medicine, Puerta de Hierro Research Institute and University Hospital, Madrid, Spain; ${ }^{3}$ Department of Pharmacology and Experimental Neuroscience, College of Medicine, University of Nebraska Medical Center, NE, USA; ${ }^{4}$ Clinical Research Laboratory, Hospital Isabel Zendal, Madrid, Spain; ${ }^{2}$ nfectious Diseases Unit, Hospital Son Llàtzer, Mallorca, Spain

\begin{abstract}
Vaccines and antivirals are the classical weapons deployed to contain, prevent, and treat life-threatening viral illnesses. Specifically, for SARS-CoV-2 infection, vaccines protect against severe COVID-19 disease manifestations and complications. However, waning immunity and emergence of vaccine escape mutants remains a growing threat. This is highlighted by the current surge of the omicron COVID-19 variant. Thus, there is a race to find treatment alternatives. We contend that oral small molecule antivirals that halt SARSCoV-2 infection are essential. Compared to currently available monoclonal antibodies and remdesivir, where parenteral administration is required, oral antivirals offer treatments in an outpatient setting with dissemination available on a larger scale. In response to this need at 2021's end, regulatory agencies provided emergency use authorization for both molnupiravir and nirmatrelvir. These medicines act on the viral polymerase and protease, respectively. Each is given for 5 days and can reduce disease progression by $30 \%$ and $89 \%$, respectively. The advent of additional oral antivirals, the assessment of combination therapies, the formulation of extended-release medications, and their benefit for both early treatment and prophylaxis will likely transform the landscape of the COVID-19 pandemic.
\end{abstract}

\section{Keywords}

Molnupiravir. Nirmatrelvir. Oral Remdesivir. Antiviral Therapy. Combination Therapy. SARS-CoV-2. Long-Acting Antivirals. Prophylaxis. Protease Inhibitors. Mutagenesis.

\section{Introduction}

The unprecedented pandemic caused by SARS-CoV-2 infection has abruptly and significantly redirected the social, economic, scientific, and health-care landscape. Globally, more than 6 million deaths have been linked to COVID-19. Early efforts to develop and test antivirals, to prevent or treat the coronavirus, have had mixed

Correspondence to:

*Vicente Soriano

E-mail: vicente.soriano@unir.net results and disease outcomes. Studies have focused principally on drug repurposing designed to identify agents that target distinct viral life cycle components, generally testing laboratory cell-based activity against SARS-CoV-2 $2^{1,2}$. However, such early screenings have not uniformly predicted the more complex physiologic and pathogenic disease events as have been seen during natural human infections. In one among many

Received in original form: 03-01-2022

Accepted in final for: $07-01-2022$

DOI: 10.24875/AIDSRev.22000001 
examples, viral entry studies uncovered agents affecting endosomal pathways but not fusion (for example, hydroxychloroquine) and ultimately resulted in lack of efficacy in clinical settings ${ }^{3}$.

Human viral infections commonly lead to acute selflimited or life-threatening infections. Alternatively, they can become chronic and debilitating. Clinical manifestations associated with either generally require broad clinical approaches. Notably, for SARS-CoV-2, the majority of infected persons recover completely without therapy. This is particularly true among the youngest population who generally remain asymptomatic ${ }^{4}$. Beginning therapy after patients become incapacitated with end-organ disease is commonly ineffective. The need for early therapy is highlighted by the fact that antivirals are used as a prophylaxis to prevent infection, especially in persons with high risk for severe disease or in acquiring infection de novo. Thus, the greatest efficacy of antiviral agents is shown when given early during the disease course. This has already been affirmed for influenza and the human immunodeficiency virus type 1 (HIV-1) infections $^{5,6}$. Indeed, at late stages of SARS-CoV-2 disease, antiviral efficacy wanes and treatment strategy become focused only on modulating the later sequelae of infection. The drugs that show some efficacy include corticosteroids, anticoagulants, and immune modulatory agents. Therefore, initiation of antiviral treatments needs to begin as soonas possiblefollowing the diagnosis of infection, especially in those at high risk for severe illness ${ }^{7,8}$.

Herein, we outline antiviral therapy as an alternative to vaccination for treatment and prevention of SARSCoV-2 infection. This is highlighted through new small molecules with avid activities against the virus. A few medications have recently been developed to combat SARS-CoV-2 that has recently received emergency use authorization including polymerase and protease inhibitors (Table 1). While the past reports on this topic have covered compounds that inhibit viral entry, including monoclonal antibodies, and repurposed drugs that interfere with host processes required for SARSCoV-2 replication, a dearth of new information is available on viral protease and polymerase inhibitors ${ }^{9,10}$. This addition to the literature is timely and of critical need as per the requirement for parenteral administration, high cost and limited efficacy against distinct viral variants preclude a broader use of prior therapeutics.

\section{SARS-CoV-2 life cycle}

Coronaviruses are enveloped positive-sense singlestranded RNA viruses enclosed by a capsid that in- cludes the spike (S) protein that is responsible for viral entry into cells and the morphological appearance of the virion crown. Cellular entry of coronaviruses, such as SARS-CoV-2, occurs primarily through this $S$ protein that binds to the host angiotensin-converting enzyme 2 receptor. Once the nucleic acid is released within the cytosol, it is translated to viral proteins, including the polymerase that copies RNA molecules and continues the cycle of viral replication. Ultimately, thousands of virions assemble and are then released from the infected cell (Fig. 1).

Two-thirds of the SARS-CoV-2 genome is responsible for the synthesis of two polyproteins called 1a and $1 \mathrm{ab}$ (Fig. 2). Both code for the two proteases, the papain-like protease ( $P L^{\text {pro }}, n s p 3$ ) and the major protease (M $\left.^{\text {pro }}, \mathrm{nsp5}\right)^{11}$. The larger polyprotein 1ab contains 16 non-structural proteins, including the RNA-dependent RNA polymerase (RdRp, nsp12) and the exonuclease (nsp14). The RdRp functions with two essential cofactors, nsp7 and nsp8 ${ }^{12}$. In general, any inactivation of either RdRp or proteases leads to the attenuation of viral RNA synthesis.

In contrast to most RNA viruses, the large 30,000 nt coronaviruses genome encodes an RNA proofreading 3'-5' exonuclease, by its nsp 14, that increases the RNA copy fidelity ${ }^{13,14}$. Ultimately, it maintains genetic stability of the coronavirus genome ${ }^{15}$. RNA viruses with smaller genomes, such as HIV or hepatitis C virus $(\mathrm{HCV})$, do not have a reflective exonuclease. The genetic variability of coronaviruses, including SARSCoV-2, is large enough to behave as a dynamic quasispecies within each infected individual ${ }^{14,15}$.

Notably, all SARS-CoV-2 polymerase inhibitors require intracellular activation to their nucleoside triphosphate (NTP) metabolites, which act as competitive alternative substrates for the viral RdRp and are subsequently incorporated into the nascent viral RNA. The metabolic pathways, leading to the production of NTP metabolites, are cell-type dependent and differ for each molecule, as do their mechanisms of viral RNA synthesis inhibition ${ }^{10,15}$. Once incorporated into viral RNA, the metabolites of remdesivir and AT-527 cause delayed RNA chain termination. In contrast, those of molnupiravir and favipiravir act as specific viral RNA mutagens. This is due to their capability of promiscuous base pairing which ultimately lead the viral progeny to extinction throughout "error catastrophe",,10.

SARS-CoV-2 protease inhibitors bind covalently to the enzyme, acting as competitive substrates. The $\mathrm{M}^{\text {pro }}$ protease functions as a homodimer of 306 amino acids. The catalytic site contains cysteine residues. 
Table 1. Oral antivirals against SARS-CoV-2

\begin{tabular}{|c|c|c|c|c|}
\hline Drug & Viral target & Chemistry & Mechanism of action & Comments \\
\hline Molnupiravir & $\mathrm{RdRp}$ & $\begin{array}{l}\text { Cytidine } \\
\text { analog }\end{array}$ & Mutagenesis & $\begin{array}{l}\text { Reduce hospitalizations and deaths by } \\
30 \% \text { in outpatients. Potential teratogenicity }\end{array}$ \\
\hline Nirmatrelvir & $M^{\text {pro }}$ & $\begin{array}{l}\text { Small } \\
\text { molecule }\end{array}$ & Protease inhibitor & $\begin{array}{l}\text { Reduce hospitalizations and deaths by } \\
89 \% \text { in outpatients. Potential for drug } \\
\text { interactions due to ritonavir boosting. }\end{array}$ \\
\hline $\begin{array}{l}\text { GS-621763 } \\
\text { (oral remdesivir) }\end{array}$ & $\mathrm{RdRp}$ & $\begin{array}{l}\text { Adenosine } \\
\text { analog }\end{array}$ & Chain terminator & On clinical development \\
\hline AT-527 & $\mathrm{RdRp}$ & $\begin{array}{l}\text { Guanosine } \\
\text { analog }\end{array}$ & Chain terminator & On clinical development \\
\hline GC-376 & $\mathrm{M}^{\text {pro }}$ & $\begin{array}{l}\text { Small } \\
\text { molecule }\end{array}$ & Protease inhibitor & On clinical development \\
\hline PBI-0451 & $M^{\text {pro }}$ & $\begin{array}{l}\text { Small } \\
\text { molecule }\end{array}$ & Protease inhibitor & On clinical development \\
\hline
\end{tabular}

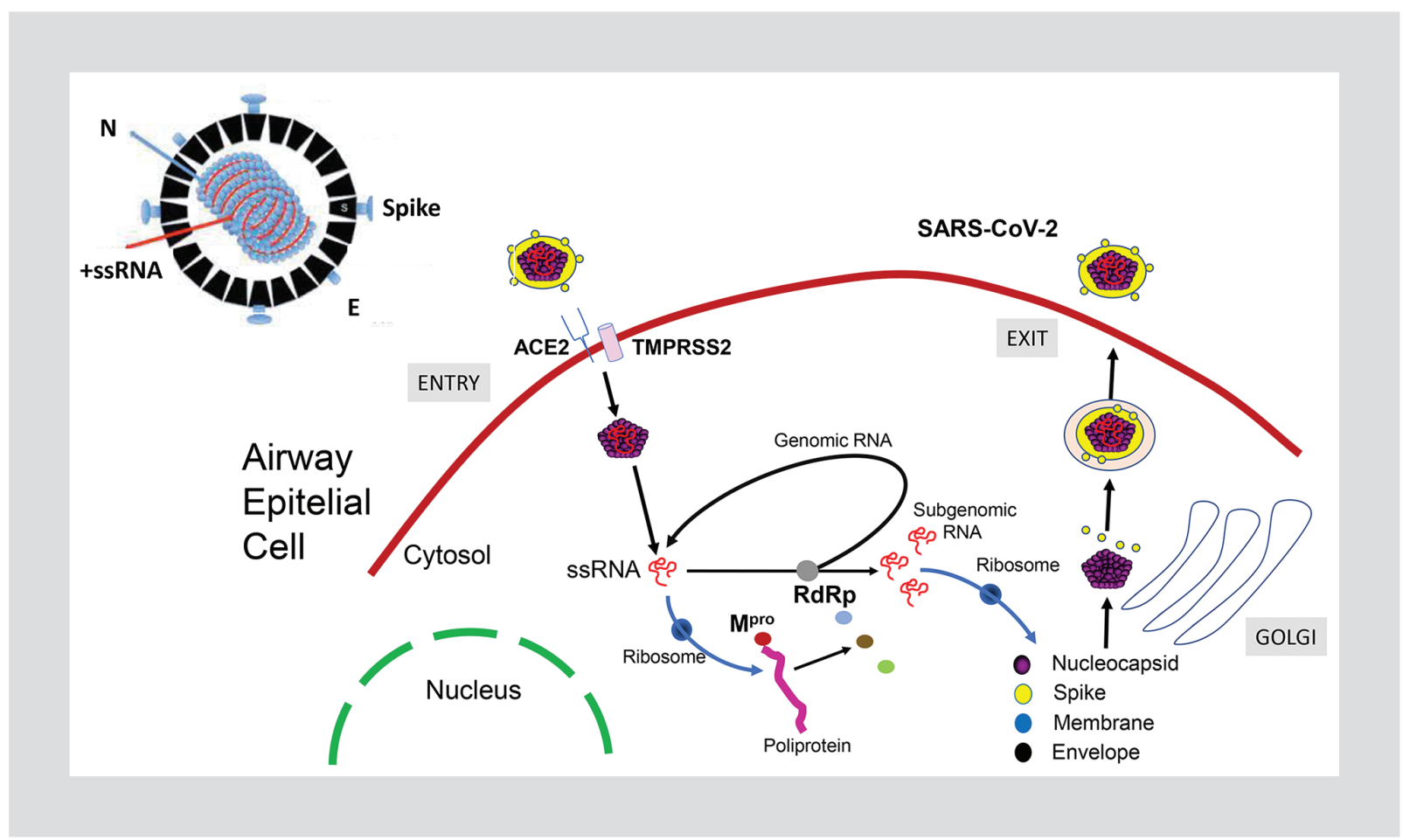

Figure 1. SARS-CoV-2 life cycle.

In contrast, the catalytic site of the second SARSCoV-2 protease ( $P L^{\text {pro }}$ ), which is larger and more complex, contains serine residues. Predicting drug susceptibility responses from viral protease geno- types could accelerate drug development based on prediction models ${ }^{16}$, as it has previously been made using other related proteases, such as the HIV-1 aspartic protease. 


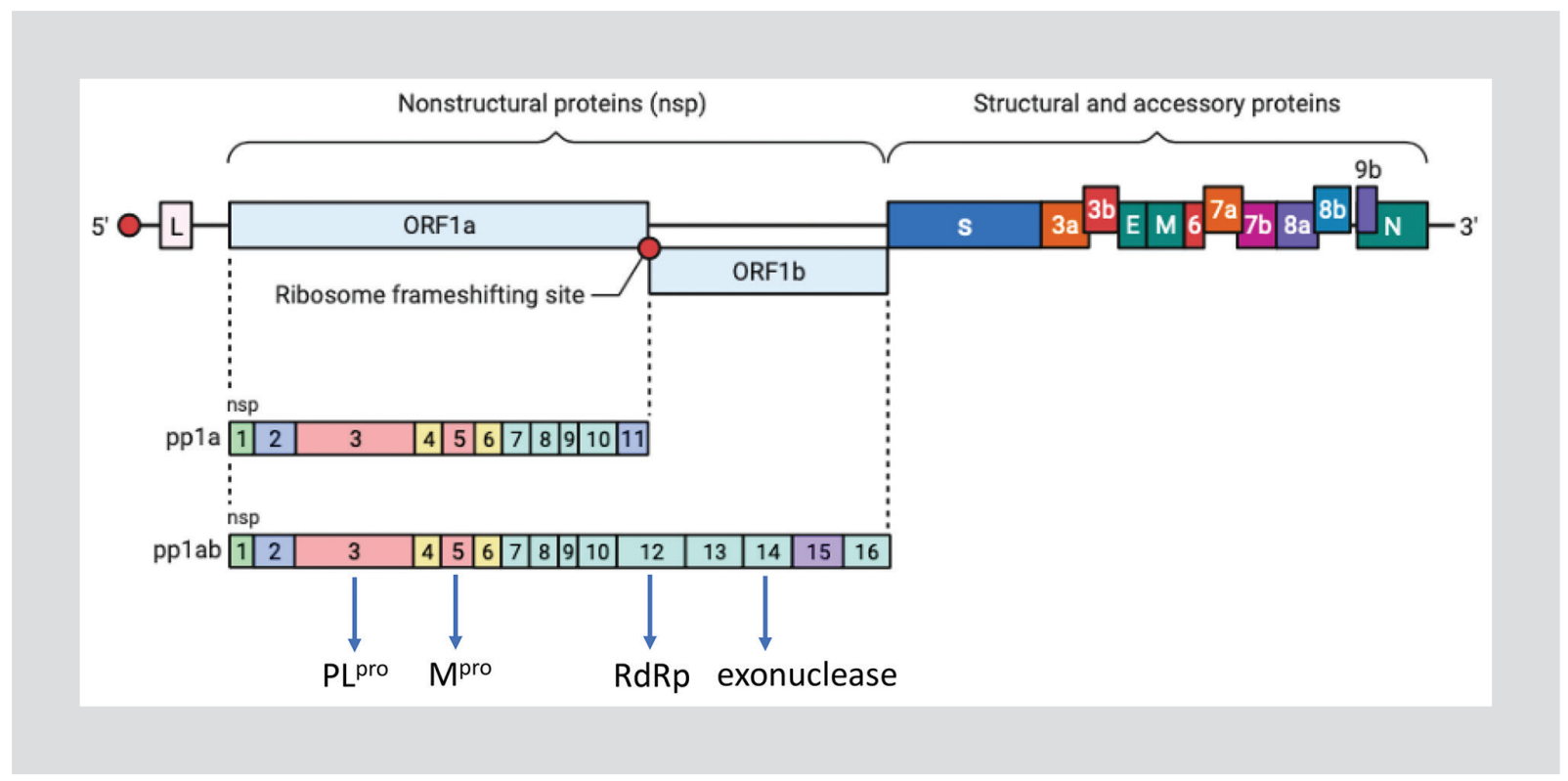

Figure 2. SARS-CoV-2 genome structure.

\section{Polymerase inhibitors}

Among RNA viruses, only a single gene product is shared between all known members, the RdRp ${ }^{17}$. Thus, it serves as a logical target for drug discovery, mostly testing nucleotide analog inhibitors. In addition, the production of RNA from an RNA template is not a catalytic activity found in human polymerases, limiting the potential to affect any native cellular processes when targeting the viral protein. However, the structure of RdRp enzymes or enzyme complexes can differ significantly among distinct RNA viruses, such as hepatitis $A$, polio, Ebola, hepatitis $C$, and influenza ${ }^{18}$. Given this wide variability between $\mathrm{RdRp}$, most studies that have tested antivirals developed to treat other RNA viruses have failed when tried against SARS-CoV-2.

Another consideration for almost all RNA viruses compared to DNA viruses refers to their relatively high error rate, partially due to a "sloppy" RdRp and generally a lack of proofreading capability. The mutagen capacities are highlighted by the flaviviruses ${ }^{19}$. This demonstrates the high degrees of diversity and, in turn, adaptability. There are few exceptions, including coronaviruses, that possess a proofreading 3' to 5' exonuclease and that ensure a minimum copy fidelity for their long genome ${ }^{18}$. This unique exonuclease activity for coronaviruses makes antiviral drug discovery challenging.

Molnupiravir is an orally bioavailable prodrug behaving as a cytidine analog. It was developed at the Em- ory University as part of a program against Venezuelan equine encephalitis virus. In preclinical models, the drug showed potent antiviral activity against both influenza and respiratory syncytial viruses. As SARS-CoV-2 emerged, molnupiravir testing showed antiviral activity in the laboratory setting and in animal models ${ }^{20,21}$.

The mechanism of the action of molnupiravir does not involve the termination of the nascent RNA chain but the introduction of wrong base pairs, causing hypermutagenesis ${ }^{22,23}$. Fortunately, molnupiravir is able to evade the SARS-CoV-2 exonuclease repairing action $^{24}$. This is in contrast with other antivirals that work similarly, such as ribavirin ${ }^{25}$.

Molnupiravir potently inhibits the replication of SARSCoV-2 in human airway cells. Recent results of a phase 2a trial have demonstrated that as the first oral, directacting antiviral, molnupiravir is highly effective in reducing nasopharyngeal SARS-CoV-2 infectious virus and viral RNA levels. Moreover, it has a favorable safety and tolerability profile 26,27 . In the MOVe-OUT Phase 3 trial, oral molnupiravir 800 mg BID was effective in reducing hospitalizations and deaths by $30 \%$, without identified safety concerns when initiated within 5 days after the onset of signs or symptoms. These results were recorded in a population of non-hospitalized, unvaccinated adults who were at risk for progression to severe disease ${ }^{28}$. Based on these data, the drug was recently approved for emergency use. It is given as $800 \mathrm{mg}$ twice daily for 5 days. The price for each course of treatment has been established at or 
around 700 US dollars. Concerns about the mutagenic effects of molnupiravir on human DNA have been highlighted, and the drug is currently contraindicated in pregnant women.

\section{GS-621763}

It is an oral prodrug of a remdesivir derivate being developed as a treatment of SARS-CoV-2 infection. Its activity has already been demonstrated in animal models ${ }^{29}$. Remdesivir was the first antiviral approved to treat severe COVID-19. Its wider use is limited by intravenous delivery. It is a prodrug of an adenine nucleoside analog that causes delayed chain termination of the nascent RNA chain. Remdesivir exhibits a broad spectrum of activity against multiple RNA viruses. It was first developed to treat hepatitis $C$ and Ebola. Interestingly, it eludes the coronavirus exonuclease repair action. As prove of its inhibitory activity, remdesivir resistance has arisen during prolonged in vitro passages ${ }^{30}$.

In humans, remdesivir is administered intravenously with a loading dose of $200 \mathrm{mg}$ followed by $100 \mathrm{mg}$ daily for 5-10 days. In registrational trials, patients receiving the drug had a median recovery of 10 days versus 15 days for those in the placebo group ${ }^{31}$.

\section{AT-527}

It is a prodrug of a guanosine nucleotide analogue, developed by Atea Pharmaceuticals. AT-527 was originally developed against the hepatitis C RdRp. The drug was safe in Phases 1-2 clinical trials conducted in healthy and HCV-infected subjects. Following drug repurposing efforts, AT-527 was recently found to be active in vitro against SARS-CoV-232.

\section{Favipiravir}

Being a guanosine purine nucleotide analog, favipiravir is metabolized intracellularly into its active triphosphate metabolite. This is a potent inhibitor of a wide range of RNA viruses, including influenza, flaviviruses, and coronaviruses. Interestingly, it induces lethal mutagenesis ${ }^{33}$. An oral form of favipiravir is available. The drug is approved for the treatment of epidemic new influenza viruses in Japan and it has been used as anti-COVID-19 agent in a few low- and middle-income countries, since the beginning of the pandemic. However, the drug exhibits weak activity against SARSCoV- $2^{10}$.

\section{Protease inhibitors}

\section{Nirmatrelvir (PF-07321332)}

The SARS-CoV-2 genome encodes two polyproteins which are processed by two viral proteases, $\mathrm{PL}^{\text {pro }}$ and $\mathrm{M}^{\mathrm{pro}}$. Both proteases catalyze their own release and liberate other nonstructural proteins (Nsps) from the polyproteins, building a replicase-transcriptase complex that is vital for viral transcription and replication (Fig. 1). Both Mpro and PL pro are attractive targets for drug development ${ }^{34}$. However, the simpler structure of $M^{\text {pro }}$ has made it more appealing. Furthermore, the high level of structural conservation among the $\mathrm{M}^{\text {pro }}$ of different coronaviruses give a chance to design a pancoronavirus protease inhibitor. In addition, since Mpro has no human homologue, off side effects of inhibitors are unexpected ${ }^{35}$.

PF-00835321 and its novel designed phosphate prodrug PF-07304814 are potent inhibitors in vitro of the coronavirus $\mathrm{M}^{\text {pro. }}$. The protease inhibitor was originally designed for the treatment of SARS in 2003. However, development was halted after the epidemic ended. When SARS-CoV-2 came along and genomic analyses revealed that the virus's Mpro protein was almost identical to that from the original SARS-CoV, drug studies were resumed by Pfizer ${ }^{36}$. Preclinical studies revealed that PF-00835231 exhibits potent in vitro antiviral activity against SARS-CoV-2 with suitable pharmaceutical properties. Moreover, it showed additive/synergistic effects in combination with remdesivir ${ }^{37}$.

The oral form of intravenously administered PF07304814 was developed and named PF-07321332. The oral bioavailability of PF-07321332 is a major advantage. PF-07304814 (intravenous) and PF-07321332 (oral) have been tested in Phase $2 / 3$ clinical trials ${ }^{38}$. On November 2021, the oral trial (EPIC-HR study) was prematurely discontinued after noticing a planned interim analysis a significant benefit of treated patients versus those on placebo, in terms of lower hospitalizations and deaths: $3 / 389(0.8 \%)$ versus $27 / 385(7.0 \%)$, respectively. Adverse events did not differ significantly between groups ${ }^{39}$.

Nirmatrelvir was the first oral antiviral to receive emergency use authorization by regulatory agencies. Pfizer has marketed its protease inhibitor along with ritonavir to enhance its PK profile and half-life. The cocktail is given as three pills every $12 \mathrm{~h}$ for 5 days. Two indications are considered: early diagnosis in outpatients at risk for severe disease and post-exposure 
prophylaxis. A note of caution is needed with regard to ritonavir boosting and the potential for drug interactions, given its potent inhibition of the cytochrome P450. However, the short course of therapy (only 5 days) makes this caveat less problematic than in the HIV field, as antiretrovirals need to be given indefinitely ${ }^{40}$.

\section{GC-376}

This compound has shown antiviral activity against feline infectious peritonitis coronavirus in experimentally infected cats. GC-376 showed promising antiviral activity against SARS-CoV-2 with an $\mathrm{EC}_{50}$ of $3.37 \mathrm{nM}$. Furthermore, the drug improved survival in infected mice ${ }^{41}$. The antiviral activity of GC-376 was also shown in SARS-CoV-2-infected transgenic mice. However, it was modest consistent with its weak in vitro antiviral activity. In comparison, a GC-376 analog was recently reported to improve survival in MERS CoV-infected mice. This result suggests that the cellular antiviral activity of GC-376 against SARS-CoV-2 needs to be improved by 100 -fold to achieve the desired in vivo antiviral efficacy.

\section{PBI-0451}

Pardes Biosciences recently announced preliminary results from a Phase I trial in healthy volunteers testing a new potent inhibitor of the coronavirus $M^{\text {pro }}$ enzyme. Interestingly and in contrast with nirmatrelvir, this new molecule will not need ritonavir boosting.

\section{Combination therapy}

The experience from the HIV and HCV therapeutic fields has shown that when several antivirals become available, their combination may enhance their inhibitory effect. Accordingly, the use of fixed-dose combinations of antivirals as single pills has proven a huge benefit 42 . Thus, combination therapy should be explored as soon as possible in the treatment of SARSCoV-2 infection.

Additive/synergistic effects could be seen with drugs targeting the same viral enzyme as long as their mechanism of action differs, avoiding any potential overlapping action. This is the case for nucleoside analogues and non-nucleoside HIV reverse transcriptase inhibitors (for example, tenofovir and rilpivirine). Benefits using molecules that target the same viral enzyme and with similar mechanisms of action may also occur when drug exposures differ within distinct cell types, as shown with the NTP metabolites of some HIV nucleoside analog inhibitors (i.e., using abacavir plus lamivudine or tenofovir plus emtricitabine). However, the best way to ensure synergistic antiviral effects results from combining agents that interfere with distinct enzymes critically involved in the viral life cycle. This strategy will maximize antiviral potency and minimize the risk of selection of drug-resistant viruses. In this regard, it should be reminded that drug resistance mutations pre-exist in most populations of RNA viruses, given their quasispecies nature at any time point in a given infected individual ${ }^{43}$.

The development of a combination therapy consisting of a mutagenic agent acting on the viral polymerase (for example, molnupiravir) plus a viral protease inhibitor (for example, nirmatrelvir) is an appealing strategy that could potentially enhance treatment outcomes in SARS-CoV-2-infected persons. Given that both antivirals target distinct enzymes that are uniquely found in coronaviruses and not in human cells, adverse events due to off side effects are presumably expected to be low. However, as mentioned above, molnupiravir could readily be incorporated into the host genome.

\section{Prophylaxis}

SARS-CoV-2 vaccines have proven to be very effective in halting progression to severe COVID-19 forms and, in this way, hospitalizations and deaths. In this regard, experiences from the rollout of vaccines at nationwide country level, such as in Spain, have been impressive ${ }^{44}$. As a complementary intervention, specific SARS-CoV-2 antivirals may provide protection in individuals at high risk for contagion and/or for developing severe COVID-19. Waning of vaccine-induced immunity seems to occur uniformly beyond 6 months ${ }^{45}$. Moreover, the emergence of new viral strains might evade immunity resulting from vaccines ${ }^{46}$. Indeed, breakthrough infections have been demonstrated elsewhere, in both former vaccinated and people that had experienced natural infection episodes ${ }^{45,46}$. On the other hand, antivirals will provide an alternative option to millions of immunocompromised individuals that will respond poorly to vaccines. Finally, antiviral therapies that target conserved viral proteins are likely to be effective against future pandemic coronaviruses.

The timely initiation of antiviral therapy for SARSCoV-2 will require the widespread availability of simple, affordable, and self-administered tests for the early 
diagnosis of SARS-CoV-2 infection. Current antigen tests fit well this demand with enough good sensitivity and specificity ${ }^{47-50}$. For the subset of persons at high risk for developing severe COVID-19 infection, short courses of antivirals as post-exposure prophylaxis might be considered.

\section{Inhaled antiviral formulations}

SARS-CoV-2 enters the body and initially replicates in the nose and the upper respiratory tract. In this way, both mucosal and systemic immunities develop. With the current vaccines that are administered intramuscularly, only systemic immunity develops. They do not provide mucosal immunity and sterilizing effects, although confer protection from developing severe pulmonary disease. Attempts to administer vaccines or antivirals using the intranasal route may prove to be more effective than using systemic deliveries, such as intravenous or subcutaneous routes ${ }^{51}$. In experimental animal models, the intranasal administration of a fusion inhibitor completely protects against SARS-CoV-2 infection. This was demonstrated during close contacts between uninfected and infected animals ${ }^{52}$.

\section{Long-acting antiviral formulations}

Drug adherence represents an important component in the management of chronic illnesses. This is based on the fact that missed doses translate into poor clinical results. In other viral infections, such as HIV/AIDS, poor compliance with medications may be complicated further with the selection of drug resistance and ultimately loss of drugs for further use ${ }^{53}$. For self-limited infections, such as those caused by respiratory viruses, including SARS-CoV-2, drug adherence during a 5 -day course of oral treatment might be disregarded. However, the experience from similar short courses of oral antibiotic prescription supports that outpatients with mild bacterial infections often fail to complete the planned length of therapy. Accordingly, single dosing using high antibiotic doses is often preferred, for example, when treating urinary tract infections.

The consideration of antivirals for COVID-19 prophylaxis might awake a different scenario in which longacting medications might be considered. The experience from the HIV-1 field is very appealing. Indeed, pre-exposure prophylaxis has shown its benefit in the prevention of viral acquisition in uninfected individuals engaged in high-risk behaviors ${ }^{54}$. Recent advances in chemical pharmacology have led to the emergence of ultra-long-acting (XLA) antiviral formulations that may provide drug exposure for several months or up to 1 year ${ }^{55-59}$. The advent of these medicines might transform the way to confront new COVID-19 surges. We may envision a wide prescription of XLA antivirals as soon as SARS-CoV-2 outbreaks are recognized, prioritizing persons with higher risk exposure and disease severity ${ }^{60}$.

\section{Foreseeable future}

The expected wider use of oral antivirals to be given as soon as diagnosis is made coupled with the advent of a new generation of vaccines having a broader coverage against new variants will hopefully limit the pandemic damage ${ }^{61}$. Oral antivirals can be used together with vaccines or if vaccines are expected to perform suboptimally due to immune deficiency or are contraindicated due to comorbid conditions. Given that a growing part of the population has either been exposed to natural infection or has been vaccinated, widespread immunity against SARS-CoV-2 will further increase. Such immunity, even conferring partial protection, will contribute to ameliorate symptoms and reduce the societal and medical impact of future COVID-19 waves. The lower virulence that has already been noticed with the omicron variant supports the notion that the virus itself is likely to be shifting toward a less pathogenic form as it adapts to the human host ${ }^{62}$

In contrast to SARS-CoV outbreak in 2002 that was extinguished within 9 months and led to 8422 cases and 916 deaths $^{63}$, SARS-CoV-2 could evolve into an endemic or seasonal infection. Over the winter months, it would become similar to other respiratory tract infections that include human endemic coronaviruses, rhinoviruses, influenza, and the respiratory syncytial virus ${ }^{64}$.

\section{References}

1. Khan R, Jha R, Amera G, Jain M, Singh E, Pathak A, et al. Targeting novel coronavirus 2019: a systematic drug repurposing approach to identify promising inhibitors against 3C-like proteinase and 2'-O-ribose methyltransferase. J Biomol Struct Dyn. 2020;2:1-40.

2. Gatti M, De Ponti F. Drug repurposing in the COVID-19 era: insights from case studies showing pharmaceutical peculiarities. Pharmaceutics. 2021;13:302.

3. Hoffmann M, Mösbauer K, Hofmann-Winkler H, Kaul A, Kleine-Weber $H$, Krüger N, et al. Chloroquine does not inhibit infection of human lung cells with SARS-CoV-2. Nature. 2020;585:588-90.

4. Gandhi M, Yokoe D, Havlir D. Asymptomatic transmission, the Achilles heel of current strategies to control Covid-19. N Engl J Med. 2020; 382:22-3.

5. Patel M, Shahjin F, Cohen J, Hasan M, Machhi J, Chugh H, et al. The immunopathobiology of SARS-CoV-2 infection. FEMS Microbiol Rev. 2021;45:fuab035

6. Marik P, Iglesias J, Varon J, Kory P. A scoping review of the pathophysiology of COVID-19. Int J Immunopathol Pharmacol. 2021:35:1-16.

7. Ngo B, Marik P, Kory P, Shapiro L, Thomadsen R, Iglesias J. et al. The time to offer treatments for COVID-19. Expert Opin Investig Drugs. 2021;30:505-18 
8. Griffin D, Brennan-Rieder D, Ngo B, Kory P, Confalonieri M, Shapiro L, et al. The importance of understanding the stages of COVID-19 in treatment and trials. AIDS Rev. 2021;23:40-7.

9. Hall M. Anderson J, Anderson A, Baker D, Bradner J, Brimacombe KR et al. Report of the NIH SARS-CoV-2 antiviral therapeutics summit. J Infect Dis. 2021;224:1-21.

10. Tao K, Tzou P, Nouhin J, Bonilla H, Jagannathan P, Shafer R. SARSCoV-2 antiviral therapy. Clin Microbiol Rev. 2021;34:e00109-21.

11. Wu F, Zhao S, Yu B, Chen YM, Wang W, Song ZG, et al. A new coronavirus associated with human respiratory disease in China. Nature. 2020;579:265-9

12. Gao Y, Yan L, Huang Y, Liu F, Zhao Y, Cao L, et al. Structure of the RNA-dependent RNA polymerase from COVID-19 virus. Science. 2020;368:779-82.

13. Minskaia E, Hertzig T, Gorbalenya A, Campanacci V, Cambillau C, Canard B, et al. Discovery of an RNA virus exoribonuclease that is critically involved in coronavirus RNA synthesis. Proc Natl Acad Sci U S A. 2006:103:5108-13

14. Robson F, Khan K, Le T, Paris C, Demirbag S, Barfuss P, et al. Coronavirus RNA proofreading: molecular basis and therapeutic targeting. Mo Cell. 2020:79:710-27.

15. Domingo E, García-Crespo C, Lobo-Vega R, Perales C. Mutation rates, mutation frequencies, and proofreading-repair activities in RNA virus genetics. Viruses. 2021;13:1882

16. Liang J, Pitsillou E, Ververis K, Guallar V, Hung A, Karagiannis T. Investigation of small molecule inhibitors of the SARS-CoV-2 papain-like protease by all-atom microsecond modelling, PELE Monte Carlo simulations, and in vitro activity inhibition. Chem Phys Lett. $2021 ; 139294$

17. Pathania S, Rawal R, Singh P. RdRp (RNA-dependent RNA polymerase) a key target providing anti-virals for the management of various vira diseases. J Mol Struct. 2022:1250:131756.

18. Maheden K, Todd B, Gordon C, Tchesnokov E, Götte M. Inhibition of viral RNA-dependent RNA polymerases with clinically relevant nucleotide analogs. Enzymes. 2021;49:315-54.

19. Griesemer S, Kramer L, Van Slyke G, Pata JD, Gohara DW, Cameron CE, et al. Mutagen resistance and mutation restriction of St. Louis encephalitis virus. J Gen Virol. 2017:98:201-11.

20. Wahl A, Gralinski L, Johnson C, Yao W, Kovarova M, Dinnon KH, et al. SARS-CoV-2 infection is effectively treated and prevented by EIDD-2901. Nature. 2021;591:451-7.

21. Wang Y, Li P, Solanki K, Li Y, Ma Z, Peppelenbosch MP, et al. Vira polymerase binding and broad-spectrum antiviral activity of molnupiravi against human seasonal coronaviruses. Virology. 2021;564:33-8.

22. Malone B, Campbell E. Molnupiravir: coding for catastrophe. Nat Struct Mol Biol. 2021:28:706-11.

23. Gordon C, Tchesnokov E, Schinazi R, Götte M. Molnupiravir promotes SARS-CoV-2 mutagenesis via the RNA template. J Biol Chem. 2021:297:100770.

24. Kabinger F, Stiller C, Schmitzova J, Dienemann C, Kokic G, Hillen HS, et al. Mechanism of molnupiravir-induced SARS-CoV-2 mutagenesis. Nat Struct Mol Biol. 2021;28:740-6.

25. Soriano V. Barreiro P. Is ribavirin teratogenic in humans? No evidence so far. Drug Saf. 2017;40:1163-5.

26. Fischer W, Eron J, Holman W, Cohen MS, Fang L, Szewczyk LJ, et al. A phase 2a clinical trial of molnupiravir in patients with COVID-19 shows accelerated SARS-CoV-2 RNA clearance and elimination of infectious virus. Sci Transl Med. 2021;eabl7430

27. Painter W, Holman W, Bush J, Almazedi F, Malik H, Eraut NC, et al. Human safety, tolerability, and pharmacokinetics of molnupiravir, novel broad-spectrum oral antiviral agent with activity against SARSCoV-2. Antimicrob Agents Chemother. 2021;65:e02428-20.

28. Jayk-Bernal A, Gomes da Silva M. Musungaie D, Kovalchuk E, Gonzalez A, Reyes VD, et al. MOVe-OUT Study Group. Molnupiravir for ora treatment of Covid-19 in non-hospitalized patients. N Engl J Med 2021:2116044

29. Cox R, Wolf J, Lieber C, Sourimant J, Lin MJ. Babusis D, et al. Ora prodrug of remdesivir parent GS-441524 is efficacious against SARSCoV-2 in ferrets. Nat Commun. 2021;12:6415.

30. Szemiel A, Merits A, Orton R, MacLean OA, Pinto RM, Wickenhagen A, et al. In vitro selection of Remdesivir resistance suggests evolutionary predictability of SARS-CoV-2. PLoS Pathog. 2021;17:e1009929

31. Beigel J, Tomashek K, Dodd L, Mehta AK, Zingman BS, Kalil AC, et al. Remdesivir for the treatment of COVID-19-preliminary report. N Engl J Med. 2020;383:1813-36

32. Goog S, Westover J, Jung KH, Zhou XJ, Moussa A, La Colla P, et al AT-527, a double prodrug of a guanosine nucleotide analog, is a potent inhibitor of SARS-CoV-2 in vitro and a promising oral antivira for treatment of COVID-19. Antimicrob Agents Chemother 2021:65:e02479-20.

33. Zhao L. Zhong W. Mechanism of action of favipiravir against SARSCoV-2: mutagenesis or chain termination? Innovation. 2021;2:100165.
34. Jin Z, Du X, Xu Y, Deng Y, Liu M, Zhao Y, et al. Structure of Mpro from SARS-CoV-2 and discovery of its inhibitors. Nature. 2020;582:289-93.

35. Capasso C, Nocentini A, Supuran C. Protease inhibitors targeting the main protease and papain-like protease of coronaviruses. Expert Opin Ther Pat. 2021:31:309-24.

36. Pavan M, Bolcato G, Bassani D, Sturlese M, Moro S. Supervised molecular dynamics insights into the mechanism of action of SARS-CoV-2 main protease inhibitor PF-07321332. J Enz Inhib Med Chem. 2021;36:1646-50.

37. Dampalla C, Zheng J, Dinali-Perera K, Meyerholz DK. Post-infection treatment with a protease inhibitor increases survival of mice with a fatal SARS-CoV-2 infection. Proc Natl Acad Sci U S A. 2021;118: e2101555118

38. Owen D, Allerton C, Anderson A, Aschenbrenner L, Avery M, Berritt S, et al. An oral SARS-CoV-2 Mpro inhibitor clinical candidate for the treatment of COVID-19. Science. 2021;374:1586-93.

39. Wang Z, Yang L. In the age of Omicron variant: paxlovid raises new hopes of COVID-19 recovery. J Med Virol. 2021 [Epub ahead of print]

40. Jiménez-Nácher I, Alvarez E, Morello J, Rodriguez-Nóvoa S, de Andrés S. Soriano V. Approaches for understanding and predicting drug interactions in HIV-infected patients. Expert Opin Drug Metab Toxicol. 2011;7:457-77.

41. Cáceres C, Cardenas-Garcia S, Carnaccini S, Seibert B, Rajao DS, Wang J, et al. Efficacy of GC-376 against SARS-CoV-2 virus infection in the K18 hACE2 transgenic mouse model. Sci Rep. 2021; 11:9609.

42. Soriano V, Fernandez-Montero JV, de Mendoza C, Benitez-Gutierrez L, Peña JM, Arias A, et al. Treatment of hepatitis $C$ with new fixed dose combinations. Expert Opin Pharmacother. 2017;18:1235-42

43. Coffin J. HIV population dynamics in vivo: implications for genetic variation, pathogenesis, and therapy. Science. 1995:267:483-9.

44. Barandalla I, Alvarez C, Barreiro P, de Mendoza C, González-Crespo R, Soriano V. Impact of scaling up SARS-CoV-2 vaccination on COVID-19 hospitalizations in Spain. Int J Infect Dis. 2021;112:81-8.

45. Fakhroo A, Al Khatib H, Al Thani A, Yassine $\mathrm{H}$. Reinfections in COVID-19 patients: impact of virus genetic variability and host immunity. Vaccines. 2021:9:1168.

46. Goldberg Y, Mandel M, Bar-On Y, Bodenheimer O, Freedman L, Haas EJ, et al. Waning immunity after the BNT162b2 vaccine in Israel. N Engl J Med. 2021;385:e85

47. Scheiblauer H, Filomena A, Nitsche A, Puyskens A, Corman VM, Drosten C, et al. Comparative sensitivity evaluation for $122 \mathrm{CE}$-marked rapid diagnostic tests for SARS-CoV-2 antigen, Germany, September 2020 to April 2021. Euro Surveill. 2021;26:2100441.

48. Kanii J, Proctor D, Stokes W. Berenger BM, Silvius J, Tipples G, et al. Multicenter post-implementation assessment of the positive predictive value of SARS-CoV-2 antigen-based point-of-care tests used for screening of asymptomatic continuing care staff. J Clin Microbiol. 2021; $59 \cdot 00141121$

49. Dinnes J, Deeks J, Berhane S, Davenport C, Dittrich S, Emperador D, et al, Cochrane COVID-19 Diagnostic Test Accuracy Group. Rapid, point-of-care antigen and molecular-based tests for diagnosis of SARS-CoV-2 infection. Cochrane Database Syst Rev. 2021;3: CD013705

50. Lindner A, Nikolai O, Rohardt C, Kausch F, Wintel M, Gertler M, et al. Diagnostic accuracy and feasibility of patient self-testing with a SARS-CoV-2 antigen-detecting rapid test. J Clin Virol. 2021;141:104874.

51. Rubin R. Trying to block SARS-CoV-2 transmission with intranasal vaccines. JAMA. 2021;326:1661-3.

52. de Vries R, Schmitz K, Bovier F, Predella C, Khao J, Noack D, et al. Intranasal fusion inhibitory lipopeptide prevents direct-contact SARSCoV-2 transmission in ferrets. Science. 2021;371:1379-82.

53. Havlir D, Richman D. Viral dynamics of HIV: implications for drug development and therapeutic strategies. Ann Intern Med. 1996;124: 984-94.

54. Benítez-Gutiérrez L, Soriano V, Requena S, Arias A, Barreiro P, de Mendoza C. Treatment and prevention of HIV infection with long-acting antiretrovirals. Expert Rev Clin Pharmacol. 2018:11:507-17.

55. Kulkarni T, Bade A, Sillman B, Shetty BL, Wojtkiewicz MS, Gautam N, et al. A year-long extended release nanoformulated cabotegravir prodrug. Nat Mater. 2020;19:910-20.

56. Gautam N, McMillan JM, Kumar D, Bade AN, Pan Q, Kulkarni TA, et al. Lipophilic nanocrystal prodrug-release defines the extended pharmacokinetic profiles of a year-long cabotegravir. Nat Commun. 2021; 12:3453.

57. Cobb D, Smith N, Deodhar S, Gautam N, Shetty BL, McMillan J, et al. Transformation of tenofovir into stable ProTide nanocrystals with longacting pharmacokinetic profiles. Nat Commun. 2021:12:5458

58. Deodhar S, Sillman B, Bade A, et al. Pathways for dolutegravir transformation from a daily oral to a once-a-year parenteral medicine. CROI. 2022; in press. 
59. Soriano V, Barreiro P, de Mendoza C. Long-acting antiretroviral therapy. Nat Mater. 2020;19:826-7.

60. Soriano V, Alvarez C, Edagwa B, de Mendoza C, Montoya N, Treviño A et al. Ultra-long-acting (XLA) antivirals for chronic viral hepatitis. Int $J$ Infect Dis. 2021;114:45-50.

61. Morens D, Taubenberger J, Fauci A. Universal coronavirus vaccines-an urgent need. N Engl J Med. 2021 [Epub ahead of print]
62. Kozlov M. Omicron's feeble attack on the lungs could make it less dangerous. Nature. 2022;601:177.

63. Peiris J, Yuen K, Osterhaus A, Stöhr K. The severe acute respiratory syndrome. N Engl J Med. 2003:349:2431-41.

64. Telenti A, Arvin A, Corey L, Corti D, Diamond MS, García-Sastre A, et al. After the pandemic: perspectives on the future trajectory of COVID-19. Nature. 2021:596:495-504 